\title{
Interaction between Tetraethylammonium and Permeant Cations at the Inactivation Gate of the HERG Potassium Channel
}

\author{
Hirofumi SHIMIZU, Chikashi TOYOSHIMA*, and Shigetoshi OIKI \\ Department of Physiology, Fukui Medical University, Fukui, 910-1193 Japan; and \\ * Institute of Molecular and Cellular Biosciences, The University of Tokyo, \\ Tokyo, 113-0032 Japan
}

\begin{abstract}
The fast inactivation of the human ether-à-go-go related gene product (HERG) channel is a form of C-type inactivation and is decelerated by external tetraethylammonium (TEA) and potassium. From the time constant of inactivation, the dissociation constants of TEA $\left(K^{\mathrm{TEA}}\right)$ and potassium $\left(K^{\mathrm{K}}\right)$ to the inactivation-impeding site were evaluated. $K^{\text {TEA }}$ was found to exhibit unexpected voltage dependence: $K^{\text {TEA }}$ decreased with depolarization. This was opposite the voltage dependence of $K^{\mathrm{K}}$ on inactivation, in which permeating potassium impeded closure of the inactivation gate upon binding to a site in the pore (a "foot-in-the-door" mechanism). Further experiments on inactivation revealed anomalous mole fraction effects between permeating alkali cations and TEA, while no anomalous effects
\end{abstract}

were seen between permeating ion species $\left(\mathrm{K}^{+}\right.$, $\left.\mathrm{Rb}^{+}, \mathrm{Cs}^{+}\right)$. The results indicate that TEA and permeating ions impede inactivation through binding to different but closely interacting sites. $K^{\text {TEA }}$ was influenced by permeating ions through their bindings in the pore. As the size of the occupied ion was increased the dissociation constant of TEA to the ion-occupied pore decreased. Thus, we conclude that an ion bound to the inactivation-impeding site in the selectivity filter is located in close proximity to TEA bound at the entrance of the filter. The order of affinity of alkali cations for the inactivation-impeding site, $\mathrm{Rb}^{+}>$ $\mathrm{Cs}^{+}>\mathrm{K}^{+}$, indicated that the selectivity of the site differed significantly from permeation selectivity, $\mathrm{K}^{+}>\mathrm{Rb}^{+}>\mathrm{Cs}^{+}$. [Japanese Journal of Physiology, 53, 25-34, 2003]

Key words: anomalous mole fraction effect, ion selectivity, kinetics, selectivity filter, Xenopus oocytes.

$\mathrm{P}$ Potassium channels exhibit strict ion selectivity uncompromised by a high permeation rate, the mechanism of which has been attributed to transitions and interactions of ions in multiple sites of the selectivity filter [1]. Recently, static pictures of the distribution of ions in the pore have been revealed by X-ray crystallography of the KcsA channel [2, 3]. Studies of molecular dynamics have highlighted events occurring in the time scale of picoseconds for transitions from one site to the others [4-10]. Dynamic characteristics of the ion-binding sites of the selectivity filter will enable the gap between the microscopic static picture and macroscopic ionic flow to be bridged and provide novel insights on the mechanisms of ion permeation and selectivity. Here, we extracted the individual characteristics of permeation sites from electrophysiological measurements of ion flow. We utilized fast inactivation of the human ether-à-go-go related gene product (HERG) channel and its sensitivity to tetraethylammonium (TEA) and permeating ions to examine the properties of the external mouth of the pore.

Fast inactivation of the HERG channel occurs by a form of C-type inactivation in which permeating ions impede the closure of the inactivation gate by a foot- 
in-the-door mechanism; the inactivation gate closes upon emptying of the external filter [11-19]. Consequently, the fast inactivation gate continuously monitors the microscopic vacant status of the outer selectivity filter and actuates the gate to close, which can be evaluated by the time constant of inactivation from macroscopic measurements. TEA has been a versatile tool to explore the characteristics of potassium channels. Extracellular TEA binds to potassium channels with a high dissociation constant and blocks the potassium current [20]. Electrophysiological experiments have shown that external and internal TEA bind to the opposite ends of the selectivity filter and interact with each other through ions in the filter [21]. Studies of molecular dynamics have revealed that the stability of TEA binding depends strongly on ion distributions in the selectivity filter $[22,23]$. For potassium channels exhibiting C-type inactivation, TEA slows the inactivation rate $[11,24]$. The location of the binding site for TEA is the outer entrance of the selectivity filter, where TEA decelerates the inactivation. Thus, the dissociation constant of TEA evaluated by the deceleration effects on inactivation will reveal interactions between TEA at the entrance and ions in the permeating sites. Here, the following strategies were employed to characterize a site in the selectivity filter: The fast inactivation gate of the HERG channel was used as a sensor to detect the vacancy of a site in the selectivity filter. TEA acting from the extracelluar side was used as a reporter of ion distribution in the selectivity filter. A strategy combining these approaches has allowed us to elucidate the characteristics of the inactivation-impeding site of the selectivity filter, which exhibited a marked difference in the binding selectivity from the permeation selectivity and contributes to TEA affinity through the occupying ion species.

\section{MATERIALS AND METHODS}

Oocyte isolation and cRNA injection. Female Xenopus laevis were anesthetized in tricaine solution $(1.5 \mathrm{~g} / l)$ for $30 \mathrm{~min}$ at $4^{\circ} \mathrm{C}$. Ovarian lobes were removed through a small abdominal incision. The follicular layer was removed enzymatically for $1.5 \mathrm{~h}$ with $2 \mathrm{mg} / \mathrm{ml}$ collagenase (Sigma, St. Louis, MO, USA) in $\mathrm{Ca}^{2+}$-free Barth's solution $(88 \mathrm{mM} \mathrm{NaCl}, 1 \mathrm{mM} \mathrm{KCl}$, $2.4 \mathrm{mM} \quad \mathrm{NaHCO}_{3}, \quad 0.82 \mathrm{mM} \quad \mathrm{MgSO}_{4}$, and $5 \mathrm{mM}$ HEPES, pH 7.6). HERG cRNA was prepared from cDNA (a gift provided by G. A. Robertson, University of Wisconsin, USA) [25] with a commercial kit (mMessage mMachine, Ambion, Austin, TX, USA). One day after the isolation procedure, defolliculated oocytes (stage V-VI) were injected with HERG
cRNA ( $40 \mathrm{nl}$ of $0.05 \mathrm{ng} / \mathrm{nl}$ ). After the final collection (up to five collections), the frogs were sacrificed by an overdose of tricaine $(20 \mathrm{~g} / \mathrm{l})$.

Electrophysiology. Two-electrode voltage-clamp experiments [26] were performed on the HERG expressed Xenopus oocytes ( 2 to $6 \mathrm{~d}$ after injection) using a Dagan CA1 amplifier (Dagan Corporation, Minneapolis, IL, USA) at $23^{\circ} \mathrm{C}$. The resistance of glass pipettes (borosilicate) filled with $3 \mathrm{M} \mathrm{KCl}$ was $0.5-1.0 \mathrm{M} \Omega$. The cut-off frequency of the filter was $1 \mathrm{kHz}$ and the sampling rate was $3 \mathrm{kHz}$. The bath solution contained $100 \mathrm{mM} \mathrm{XCl}(\mathrm{X}: \mathrm{K}, \mathrm{Rb}, \mathrm{Cs}, \mathrm{Na}$, NMDG [N-methyl-D-glucamine]); $1.8 \mathrm{mM} \mathrm{CaCl}_{2}, 1$ $\mathrm{mM} \mathrm{MgCl}_{2}$, and $5 \mathrm{mM}$ HEPES, pH 7.5. Except for solutions used for mole fraction experiments ([X]+ [TEA] $=100 \mathrm{mM}$ ), NMDG was added as an inert cation. Hereafter, square brackets mean concentration. Raw two-electrode voltage clamp data were not leakage or capacitance subtracted. The current traces were fitted by an exponential function, $I(t)=A \exp \left(-t / \tau_{\text {Inact }}\right)+C$, where $A$ is an amplitude and $C$ is a constant, using pClamp (Axon Instruments, Inc., Union City, CA, USA). The reversal potential was obtained when oocytes were bathed in a $100 \mathrm{mM} \mathrm{XCl}(\mathrm{X}: \mathrm{K}, \mathrm{Rb}$, $\mathrm{Cs}, \mathrm{Na}$, and NMDG) solution. The permeability ratio $\left(P_{\mathrm{X}} / P_{\mathrm{K}}\right)$ was calculated using the Goldman-HodgkinKatz equation [1], in which intracellular potassium concentration was estimated from the reversal potential in the pure potassium solution. Data were analyzed using Origin (MicroCal Software, Inc., Northampton, MA, USA) and Mathematica (Wolfram Research, Inc., Champaign, IL, USA). All data are shown as mean \pm SE $(n=5-9)$. For the fitted parameters, error propagations were taken into account [27].

\section{RESULTS}

\section{Strange voltage dependence of the dissocia- tion constant of TEA}

The fast inactivation of the HERG channel was investigated by applying electrophysiological methods to a heterologous expression system (Xenopus oocytes). A double-pulse protocol enlightened the inactivation process (Fig. 1A): From the holding voltage of -80 $\mathrm{mV}$, a long depolarizing pulse $(3.0 \mathrm{~s})$ at $+20 \mathrm{mV}$ activated the channel, which subsequently underwent a very fast inactivation process $(\mathrm{C} \rightarrow \mathrm{O} \rightarrow \mathrm{I})$, where $\mathrm{C}, \mathrm{O}$, I represent the closed-, open-, and inactivated-state, respectively. Then, a short interpulse $(30-50 \mathrm{~ms})$ at $-100 \mathrm{mV}$ recovered the channel from inactivation ( $\mathrm{I} \rightarrow \mathrm{O}$ : deinactivation). Finally, the second depolarizing pulse, at various voltages, manifested the pure inactivation process $(\mathrm{O} \rightarrow \mathrm{I})$. The current traces for the 
A

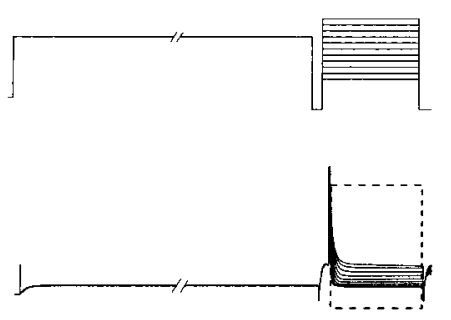

C
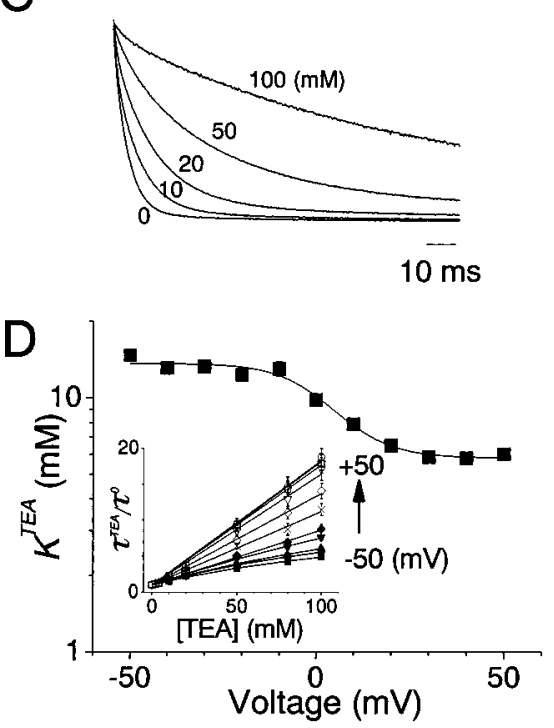

B

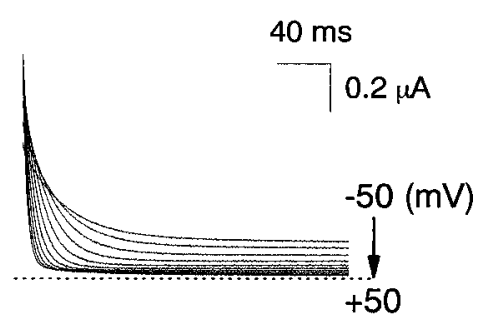

$E$
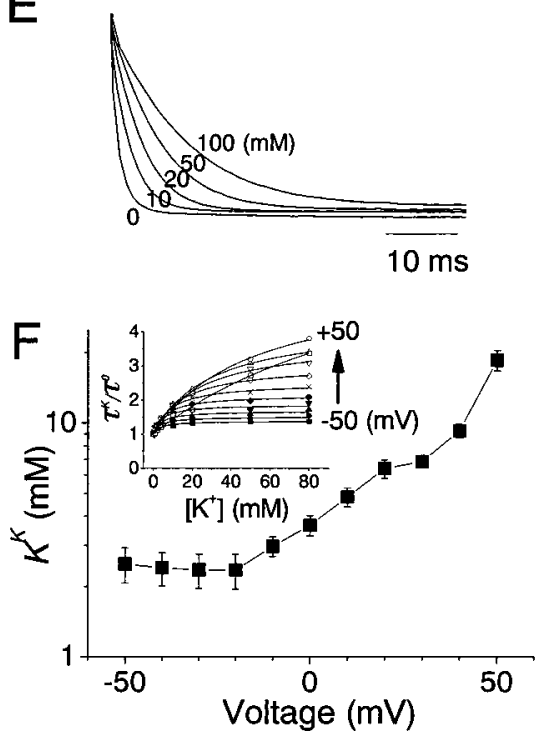

Fig. 1. Inhibitory actions of TEA and potassium on inactivation gating. A: Voltage-clamp experiments of the HERG channel expressed in Xenopus oocytes. A command pulse protocol and representative current traces in the absence of external potassium (100 mM NMDG) are shown. A double-pulse protocol was applied to investigate the inactivation process. The current traces demarcated by a broken line are expanded and shown in B. B: Inactivating current traces from -50 to $50 \mathrm{mV}$. The broken line indicates the zero current level. C, E: Current traces in the presence of external TEA (C) and potassium (E) measured at $+50 \mathrm{mV}$. The current amplitudes were normalized. D: The voltage dependency of the $K^{\text {TEA }}$ values. Inset: The concentration dependency of the $\tau^{\mathrm{TEA}} / \tau^{\circ}$ values. $\tau^{\mathrm{TEA}} / \tau^{\circ}$ was plotted as a function of TEA concentration and fitted by Eq. A1. F: The voltage dependency of the $K^{K}$ values. Inset: The concentration dependency of the $\tau_{\mathrm{K}}$ values. $\tau^{\mathrm{K}} / \tau^{\mathrm{O}}$ was plotted as a function of potassium concentration and was fitted by Eq. A2. fast inactivation are shown for the membrane potentials from -50 to $+50 \mathrm{mV}$ recorded in the absence of external potassium (Fig. 1B). As the membrane potential was depolarized, the current inactivated more rapidly and more completely.

The effect of TEA on inactivation at $+50 \mathrm{mV}$ is shown in Fig. 1C. As the TEA concentration increased, the time course of inactivation was significantly prolonged. The slower inactivation has been described for open channel blockers, where the blocked channel does not gate (inactivate) unless it is unblocked [28], which is represented by the following scheme.

$$
\mathrm{B} \underset{k_{\text {unblock }}}{\stackrel{[\mathrm{TEA}] k_{\text {block }}}{\leftrightarrows}} \mathrm{O} \underset{k_{\text {DInact }}}{\stackrel{k_{\text {Inact }}}{\rightleftarrows}} \text { । Scheme } 1
$$

where $\mathrm{B}$ represents the blocked-state; $k_{\text {Inact }}$ and $k_{\text {DInact }}$ are the inactivation and deinactivation rate constants; and $k_{\text {block }}$ and $k_{\text {unblock }}$ are the blocking and unblocking rate constants. The dissociation constant of TEA $\left(K^{\mathrm{TEA}}\right)$ is $k_{\text {unblock }} / k_{\text {block }}$.

From Scheme 1 the time constant of inactivation $\left(\tau^{\mathrm{TEA}}\right)$ can be formulated as a function of TEA concentration:

$$
\tau^{\mathrm{TEA}}=\frac{1}{k_{\text {Inact }}} \frac{1+[\mathrm{TEA}] / K^{\mathrm{TEA}}}{1+K_{\mathrm{I}}\left(1+[\mathrm{TEA}] / K^{\mathrm{TEA}}\right)}
$$

where $K_{\mathrm{I}}$ is the equilibrium constant for inactivation ( $=k_{\text {DInact }} / k_{\text {Inact }}$, all equations are listed in APPENDIX). The $K^{\mathrm{TEA}}$ value was evaluated from the data shown in Fig. 1D inset, where normalized $\tau_{\text {Inact }}\left(\tau^{\mathrm{TEA}} / \tau^{0}\right)$ was plotted for different TEA concentrations (and different membrane potentials) [11]. $\tau^{\mathrm{TEA}} / \tau^{\mathrm{O}}$ exhibited linear relationships at most of the membrane potentials tested. Thus $K_{\mathrm{I}}$ is negligible, and the slope indicates $1 / K^{\mathrm{TEA}}$. The $K^{\mathrm{TEA}}$ values for different membrane potentials are shown in Fig. 1D. Surprisingly, $K^{\mathrm{TEA}}$ decreased significantly as the membrane potential was depolarized, and the voltage-dependence of $K^{\mathrm{TEA}}$ was sigmoidal in shape. This is opposite the general effect of TEA acting from the extracellular side, in which a binding site located at the entrance of the pore is under the influence of a fraction of the membrane electric field. Thus, the opposite voltage dependence of TEA on HERG channel inactivation cannot be explained by the usual simple blocking mechanisms.

\section{Potassium inhibits inactivation by a "foot-in- the-door" mechanism}

As was the case for TEA, external potassium slowed the inactivation rate significantly (Fig. 1E). The Fig. $1 \mathrm{~F}$ inset shows that $\tau_{\text {Inact }}$ increased as the potassium concentration was increased. Upon binding to a site, a permeating ion impedes the inactivation 
gate [16] as shown in Scheme 2.

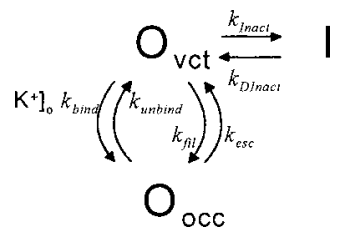

Scheme 2

where $\left[\mathrm{K}^{+}\right]_{0}$ represents the extracellular potassium concentration and $\mathrm{O}_{\mathrm{occ}}, \mathrm{O}_{\mathrm{vct}}$, and I indicate the ion-occupied open state, ion-vacant open state, and inactivated state, respectively. In this scheme, the ion-vacant state does not refer to a channel state without any ions in the pore. Rather, one of the ion binding sites, upon occupied inactivation is impeded (an inactivationimpeding site), is vacant, leaving the rest of the sites unconcerned. Here, the simplest case for the transition between two permeating states of the pore is shown. This model represents the shift model deduced from ion distribution in the KcsA potassium channel [3, 5]; $k_{\text {bind }}$ and $k_{\text {unbind }}$ are the ion-binding and ion-unbinding (from outer site) rate constants, respectively, $k_{\text {esc }}$ and $k_{\text {fil }}$ are the ion-escaping and ion-filling (from inner sites) rate constants. The steady-state probabilities of the open states are expressed using these four rate constants [29]. $K^{\mathrm{K}}$ is the dissociation constant of potassium and is given by $\left(k_{\text {unbind }}+k_{\text {esc }}\right) / k_{\text {bind }} . \tau^{\mathrm{K}}$ is expressed as follows:

$$
\tau^{\mathrm{K}}=\frac{1}{k_{\text {Inact }}^{\mathrm{o}}} \frac{1+[K] / K^{\mathrm{K}}}{1+K_{\mathrm{I}}\left(1+[\mathrm{K}] / K^{\mathrm{K}}\right)}
$$

where the apparent inactivation rate, $k_{\text {Inact }}^{0}\left(=p_{\text {vct }}^{0} \times\right.$ $\left.k_{\text {Inact }}\right)$, is the rate in the absence of external potassium.

The $K^{\mathrm{K}}$ value was evaluated from concentration dependencies of $\tau^{\mathrm{K}}$ (Eq. 2). The normalized $\tau^{\mathrm{K}}$ values $\left(\tau^{\mathrm{K}} / \tau^{\mathrm{o}}\right)$ were fitted by Eq. A2 (see APPENDIX), in which non-linearity was parameterized by the nonzero value of $K_{\mathrm{I}}$. The $K^{\mathrm{K}}$ values for different membrane potentials are shown in Fig. 1F. In contrast to TEA action, the $K^{\mathrm{K}}$ value increased significantly as the membrane potential was depolarized. Thus, the ion binding site impeding the inactivation locates in the pore under the influence of the electric field (i.e., a mechanism known as "foot-in-the-door").

\section{Interactive action of TEA and $\mathrm{K}^{+}$on HERG in- activation}

The effects of external potassium on $K^{\mathrm{TEA}}$ were examined. In Fig. 2 and inset, $K^{\text {TEA }}$ evaluated from $\tau_{\text {Inact }}$ was shown, in which the external potassium concentration was fixed. In the inset, $\tau_{\text {Inact }}$ was plotted as a function of the TEA concentration. Two sets of data for different potassium concentrations $(0$ and $20 \mathrm{mM}$

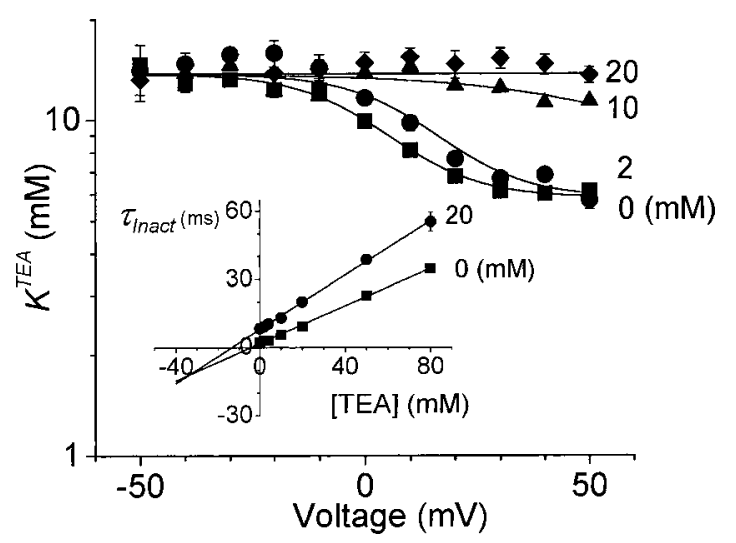

Fig. 2. Interactive inhibition of TEA and potassium on inactivation gating. The $K^{\mathrm{TEA}}$ values and its voltage and potassium dependency are shown. The $K^{\text {TEA }}$ values were obtained from the concentration dependency of the $\tau^{\mathrm{TEA}} / \tau^{\circ}$ values. $K^{\text {TEA }}$ in the presence of fixed concentrations of external potassium (0-20 mM) were plotted as a function of the voltage. The data for $0 \mathrm{mM}$ potassium was reproduced from Fig. 1D as a reference. Inset: The $\tau^{\mathrm{TEA}}$ values plotted as a function of TEA concentration measured at $+50 \mathrm{mV}$ in 0 or $20 \mathrm{~mm}$ potassium. The data were fitted by a straight line. The exptrapolated lines intersect at the third quadrant. From Eq. A5, the crossing point gave the $K_{\text {occ }}^{\mathrm{TEA}}$ value from the abscissa and the $K_{\mathrm{OCC}}^{\mathrm{TEA}} / K^{\mathrm{TEA}}$ value from the ordinate.

at $+50 \mathrm{mV}$ ) are shown. The data points were fitted by straight lines. From Eq. 1, different slopes indicate different $K^{\text {TEA }}$ values. Therefore, $K^{\text {TEA }}$ was affected by external $\mathrm{K}^{+}$. This is good evidence that TEA and potassium interact with each other in the inactivation process. It is noted that the extrapolated lines intersected at the third quadrant, which occurs between uncompetitive inhibitors (see the following section on the mole-fraction effect) [30].

Voltage dependencies of $K^{\mathrm{TEA}}$ for four different potassium concentrations are shown in Fig. 2. $K^{\text {TEA }}$ at $2 \mathrm{mM} \mathrm{K}^{+}$exhibited similar voltage dependency with a sigmoidal shape to those observed at a potassium concentration of zero. On the other hand, at $20 \mathrm{mM} \mathrm{K}^{+}$, the voltage dependency of $K^{\text {TEA }}$ was almost abolished. This sigmoidal shape and its abolition by external potassium were intriguing features of the interaction between potassium and TEA. Comparing $K^{\mathrm{TEA}}$ (Fig. 1D) with $K^{\mathrm{K}}$ (Fig. 1F) as a function of voltage, it is noted that a mirror-image relationship exists between the two dissociation constants: As the membrane potential depolarizes, the $K^{\mathrm{TEA}}$ value decreases, which coincides with the increase in the $K^{\mathrm{K}}$ value. At potentials with higher $K^{\mathrm{K}}$ values, the occupancy of potassium on the inactivation-impeding site should be lower and those channels have lower dissociation constant to TEA. Thus, it is likely that the dissociation constant of TEA is controlled by the existence of a 
potassium ion on a specific site of the selectivity filter. A plausible model of these interactions, in which TEA binds to both vacant and occupied pores, was introduced (Scheme 3).

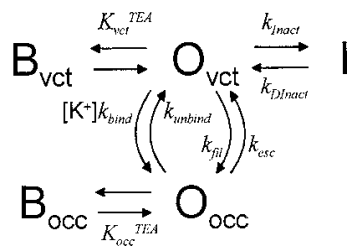

Scheme 3

where $B_{\text {vct }}$ and $B_{\text {occ }}$ represent the ion-vacant blocked state and ion-occupied blocked state, respectively, and $K_{\mathrm{vct}}^{\mathrm{TEA}}$ and $K_{\mathrm{occ}}^{\mathrm{TEA}}$ are the dissociation constants of TEA to the vacant and occupied pores. $\tau_{\text {Inact }}$ is expressed by Eq. A3 (see APPENDIX).

\section{Anomalous mole-fraction effect between per- meating ions and blocking TEA}

To elucidate the mechanism of interaction between two inhibitors, mole fraction experiments were performed. In the Fig. 3A inset, current traces recorded at $+50 \mathrm{mV}$ in the pure $\left(\mathrm{K}^{+}\right.$or TEA) and mixed $\left(\mathrm{K}^{+}\right.$and TEA) external solutions are shown. The time course of inactivation in the $1: 1$ mixed solution was not between those in two pure solutions. In the figure, the concentration dependency of $\tau_{\text {Inact }}$ in the pure potassium solution (the open triangle symbol) is shown as a reference, as well as the pure TEA effect plotted in the opposite direction of the abscissa (the open square symbol). In mole fraction solutions, the $\tau_{\text {Inact }}$ values deviated upward significantly from the linear relationship that is roughly a sum of the pure effects, and showed the maximum value in the mixed solution (Fig. 3A). This anomalous mole-fraction effect indicates that TEA and potassium bind to different sites and impede inactivation.

The anomalous mole-fraction effect was analyzed quantitatively by fitting the data to Eq. A3, where the parameters obtained hitherto were used for new parameter $\left(K_{\text {occ }}^{\mathrm{TEA}}\right)$ fitting. From the fitted parameters, hypothetical lines were drawn for competitive and uncompetitive actions. In the figure, the straight broken line indicates a competitive action (TEA does not bind to the ion-occupied pore; $K_{\mathrm{occ}}^{\mathrm{TEA}}=\infty$ ) and the curved broken line indicates an uncompetitive action (TEA can bind to the channel with the same dissociation constant whether a site in the pore is occupied by an ion or not; $\left.K_{\mathrm{occ}}^{\mathrm{TEA}}=K_{\mathrm{vct}}^{\mathrm{TEA}}\right)$. The data points fall between the competitive and uncompetitive lines, indicating that $K_{\text {occ }}^{\mathrm{TEA}}$ is not equal to but larger than $K_{\mathrm{vct}}^{\mathrm{TEA}}\left(\infty \gg K_{\text {occ }}^{\mathrm{TEA}}>\right.$ $K_{\text {vct }}^{\mathrm{TEA}}$ ). A similar result is shown in Fig. 2 and its inset: $K^{\mathrm{TEA}}$ increased as the fixed concentration of potassium was raised. This happens only when $K_{\mathrm{occ}}^{\mathrm{TEA}}>K_{\mathrm{vct}}^{\mathrm{TEA}}$ (see Eqs. A4 and A5). Both experiments indicate that TEA binding to the ion-occupied pore is weaker than that to the vacant pore.

We also used other alkali cations. Outward currents at positive potentials were little affected by $\mathrm{Rb}^{+}$and $\mathrm{Cs}^{+}$, and inactivating currents were recorded at +50
A

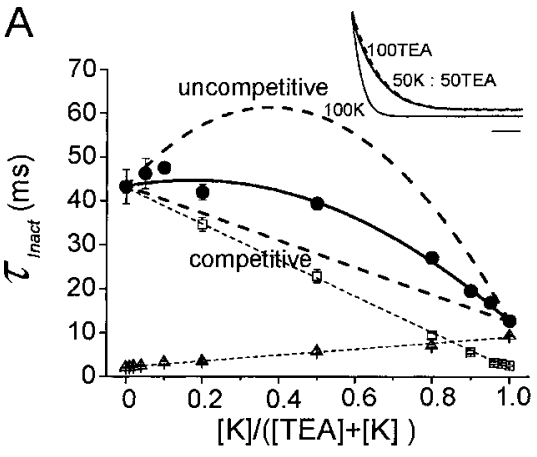

C

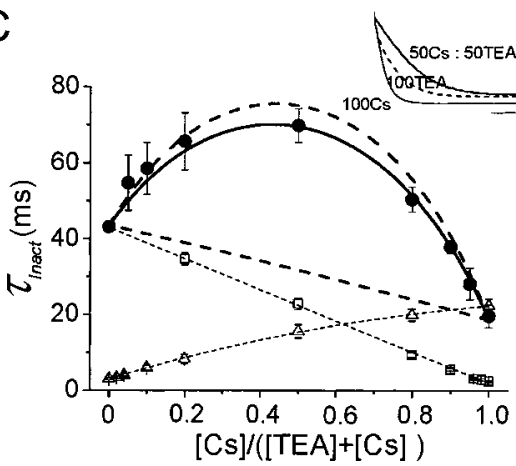

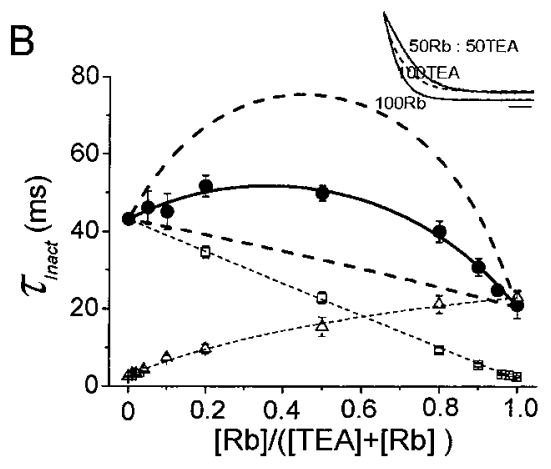

D

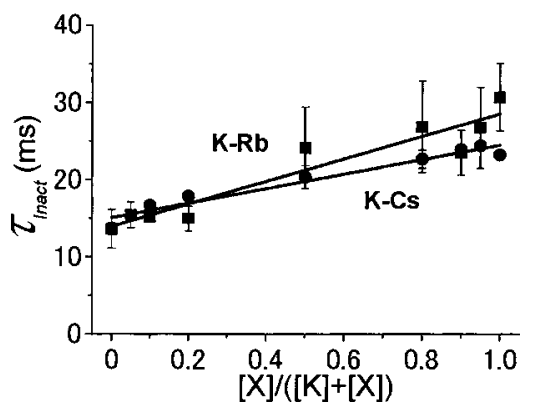

Fig. 3. Mole fraction experiments for permeating ions and TEA. A: Mole-fraction effects of external potassium and TEA on $\tau_{\text {Inact. }}$ The $\tau_{\text {nact }}$ values (filled circles; at $+50 \mathrm{mV}$ ) were plotted as a function of mole fractions $([\mathrm{K}]+$ $[T E A]=100 \mathrm{~mm})$. The data were fitted by Eq. A3 with the fitted $K_{\text {occ }}{ }^{\text {TEA }}$ value of $20.7 \pm 3.97 \mathrm{mM}(n=5)$. The broken lines indicate competitive (linear) and uncompetitive (curved) inhibition. Open symbols indicate the $\tau_{\text {Inact }}$ values in pure solutions, where the abscissa should read $[K] / 100$ for the triangle symbol and 1-[TEA]/100 for the square symbol. Inset for A-C: Normalized current traces for inactivation at $+50 \mathrm{mV}$ in the mole fractions of $0,0.5$, and 1 . The scale bar indicates $50 \mathrm{~ms}$. B: Mole-fraction effects of Rb-TEA for $\tau_{\text {nnact }}$. The fitted $K_{\text {occ }}^{\text {TEA }}$ value was $14.9 \pm 2.55 \mathrm{mM}(n=5)$. C: Mole-fraction effects of Cs-TEA for $\tau_{\text {Inact }}$. The fitted $K_{\text {occ }}^{\text {TEA }}$ value was $8.8 \pm 1.15 \mathrm{mM}$ $(n=5)$. D: Mole-fraction effects of alkali cations (K-Rb and K-Cs) on $\tau_{\text {nact }}$. 
$\mathrm{mV}$. As shown in Fig. 3B and $\mathrm{C}$, inactivation was slowest for the mixed solution of an alkali cation and TEA. Prominent anomalous mole-fraction effects on $\tau_{\text {Inact }}$ were seen for $\mathrm{Cs}^{+}$, where data points approached the uncompetitive curve. $\mathrm{Rb}^{+}$and TEA also exhibited an anomalous effect. In this case, the data points located at the middle of the competitive and uncompetitive lines. In contrast to the interaction between ions and TEA, mole fraction experiments for two species of alkali cations $\left(\mathrm{K}^{+}\right.$vs. $\mathrm{Rb}^{+}$and $\mathrm{K}^{+}$vs. $\left.\mathrm{Cs}^{+}\right)$showed no anomalous behavior (Fig. 3D). The results indicate that permeating ions compete for a site in the pore. These mole fraction experiments revealed that interaction between TEA and ions depends largely on ion species and that the inactivation-impeding site for permeating ions is localized rather than spread over the selectivity filter.

Anomalous mole-fraction effects for different ion species were analyzed quantitatively. We focused on the dissociation constant of TEA to the ion-occupied pore, $K_{\text {occ }}^{\mathrm{TEA}}$, since it reflects the pattern of the data points and is sensitive to ion species. The $K_{\text {occ }}^{\mathrm{TEA}}$ value was the largest for the $\mathrm{K}^{+}$-occupied pore and decreased as the ionic radius of the occupied ion increased $\left(K_{\text {occ }}^{\mathrm{TEA}}\right.$ for $\mathrm{K}^{+}(1.33 \AA)=20.7 \pm 3.97 \mathrm{mM}$; for $\mathrm{Rb}^{+}(1.48 \AA)=14.9 \pm 2.55 \mathrm{mM}$; for $\mathrm{Cs}^{+}(1.69 \AA)=8.8 \pm$ $1.15 \mathrm{mM}(n=5))$. How do such small differences in the ionic radii reflect on the sizable differences in the dissociation constant of TEA? Larger ions do not become an obstacle for TEA binding, but rather increases the affinity. Possible mechanisms will be discussed.

\section{Ion specificity of the ion-binding site imped- ing inactivation}

Binding of the alkali ions alone to the inactivationimpeding site in the selectivity filter was evaluated from the concentration-dependence of the $\tau_{\text {Inact }}$ values at $+50 \mathrm{mV}$ (Fig. $3 \mathrm{~A}-\mathrm{C}$ ). The $K^{\text {Ion }}$ values for alkali cations are shown in Table 1 . The selectivity of the inactivation-impeding site was Eisenman's sequence II $\left(\mathrm{Rb}^{+}>\mathrm{Cs}^{+}>\mathrm{K}^{+}\right)[1]$. As a reference, the permeability coefficients were obtained from the reversal potentials under bi-ionic conditions (see METHODS) and the permeability ratio is shown in Table 1 . The selectivity sequence was: $\mathrm{K}^{+}>\mathrm{Rb}^{+}>\mathrm{Cs}^{+}$. A discrepancy appeared between the affinity to the inactivation-impeding site and permeation selectivity. Thus, a local property of the inactivation-impeding site was extracted from the multiple ion-binding sites in the selectivity filter.
Table 1. Ion selectivity for the inactivation-impeding site and throughput permeation.

\begin{tabular}{lccc}
\hline & $\mathrm{K}^{+}$ & $\mathrm{Rb}^{+}$ & $\mathrm{Cs}^{+}$ \\
\hline$K^{\text {lon }}(\mathrm{mM})$ & $18.6 \pm 1.86$ & $5.49 \pm 0.80$ & $8.22 \pm 1.18$ \\
$P_{\mathrm{X} / P_{\mathrm{K}}}$ & 1 & $0.95 \pm 0.02$ & $0.24 \pm 0.01$
\end{tabular}

The dissociation constants of alkali cations to the inactivation-impeding site were evaluated from the data shown in Fig. 3 (open symbols) $(n=5)$. The permeability ratios $\left(P_{\mathrm{X}} / P_{\mathrm{K}}\right)$ were calculated from the reversal potentials $(n=10)$.

\section{DISCUSSION}

In this paper, we have investigated the action of TEA and permeant ions interacting with the inactivation gate and obtained a detailed picture of the inactivation-impeding site of the HERG channel. TEA and an alkali cation bind to different sites and both impede inactivation. The binding site of TEA should locate at the entrance of the pore, since the voltage-dependence of $K^{\mathrm{TEA}}$ is eliminated as the potassium concentration is raised (Fig. 2). On the other hand, the binding site of the permeating ions is located in the pore, which is indicated by voltage-dependent $K^{\mathrm{K}}$. The mole fraction experiments between alkali cations revealed that the inactivation-impeding action of permeating ions is competitive. In the selectivity filter, inactivation is impeded by an ion bound to a localized site.

Recent progress on the permeation mechanism in the KcsA channel assumes that permeating ions move in the selectivity filter in a concerted manner: The predominant ion distribution in the selectivity filter has been proposed to be $\mathrm{W}-\mathrm{I}-\mathrm{W}-\mathrm{I}$ or $\mathrm{I}-\mathrm{W}-\mathrm{I}-\mathrm{W}$ for four binding sites (from the extracellular side) where I represents a permeating ion and $\mathrm{W}$ represents a water molecule. Ion permeation operates preferentially by transition between these two distributions [3, 5]. Here, two permeation states in our permeation-coupled inactivation scheme (Scheme 3 ) are allocated to the ion distributions in the shift model (Fig. 4). In our experiments, the results plotted in Fig. 1F show that raising external concentration decelerates inactivation. In the shift model, increased external potassium boosts the shift in the distribution from $\mathrm{W}-\mathrm{I}-\mathrm{W}-\mathrm{I}$ to $\mathrm{I}-\mathrm{W}-\mathrm{I}-\mathrm{W}$. Thus, our results, based on the shift model, reveal that inactivation proceeds from $\mathrm{W}-\mathrm{I}-\mathrm{W}-\mathrm{I}$ distribution and W-I-W-I corresponds to the "vacant" state (Fig. 4).

TEA binds more tightly to the vacant pore than to the occupied pore, thus, the apparent dissociation constant of TEA reflects the vacant probability of the pore. Lower $K^{\mathrm{TEA}}$ values at depolarized potentials with their sigmoidal voltage dependency (Fig. 2) indi- 


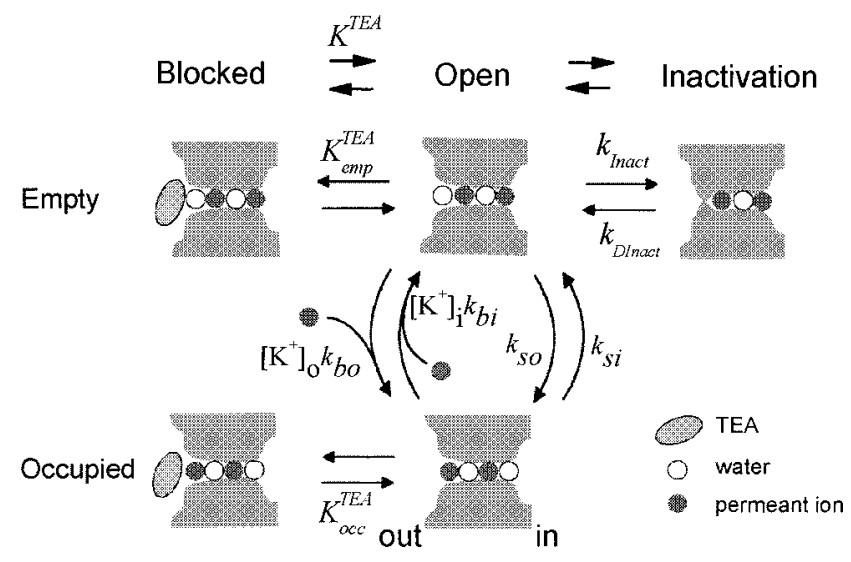

Fig. 4. A model for interaction among permeating ions, TEA, and the inactivation gate. This model implements the permeation mechanism of the shift model into the vacancy-coupled inactivation scheme (Scheme 3). Permeation occurs through transition between two ion distributions (W-I-W-I and $|-W-|-W)$. External potassium shifts the distribution from $\mathrm{W}-\mathrm{I}-\mathrm{W}-\mathrm{I}$ to $\mathrm{I}-\mathrm{W}-\mathrm{I}-\mathrm{W}$ with the rate of $\left[\mathrm{K}^{+}\right]_{\mathrm{o}} k_{\mathrm{bo}}$, where $k_{\mathrm{bo}}$ is the binding (second-order) rate constant from the extracellular side. The opposite shift occurs by intracellular potassium with the rate of $\left[\mathrm{K}^{+}\right]_{\mathrm{i}} k_{\mathrm{bi}}$, where $k_{\mathrm{bi}}$ is the binding rate constant from the inside. Transition between two ion distributions also takes places without the contribution of ions outside the selectivity filter. The firstorder rate constants for the transition are $k_{\text {so }}$ and $k_{\text {si }}$.

cate that vacant probability increased with depolarization. This was true even in the absence of external potassium. The site should be filled by potassium from the inner sites, and hyperpolarization facilitates the filling. This seemingly opposite voltage dependency is explained in the shift model (Fig. 4) if the intracellular potassium association rate $\left(\left[\mathrm{K}^{+}\right]_{\mathrm{i}} k_{\mathrm{bi}}\right)$ overwhelms the transition rate from $\mathrm{I}-\mathrm{W}-\mathrm{I}-\mathrm{W}$ to $\mathrm{W}-\mathrm{I}-$ $\mathrm{W}-\mathrm{I}\left(k_{\mathrm{si}}\right)$. The shift model also supports the high voltage dependence of $K^{\mathrm{K}}$, since permeating potassium ions reach the inactivation-impeding site through concerted movements with larger effective charge. The shift model simplified the permeation issues and aided in the interpretation of our experimental data.

The inactivation-impeding site in the "Shaker" potassium channel has been proposed to be located in the outer selectivity filter [16]. By assuming an exclusive shift model for the permeation mechanism, we concluded that the $\mathrm{W}-\mathrm{I}-\mathrm{W}-\mathrm{I}$ distribution is the "vacant" state. If an ion occupies either the first site or the third site of the selectivity filter, conformational change for inactivation cannot be initiated. We found that the binding of TEA is governed by the ion species occupied in the pore, with the dissociation constant being decreased as the size of the occupied ion is increased. Such ion-size sensitivity and decremental size-dependency strongly suggest that the inactiva-

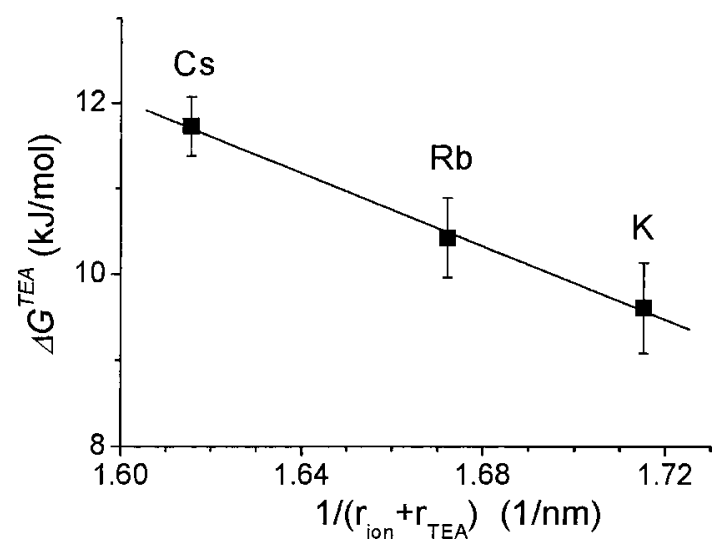

Fig. 5. TEA binding energy and ionic radius occupied in the pore. The free energy $(\Delta G)$ of the TEA binding to the occupied pore as a function of an inverse of the distance between an ion and TEA $\left(r_{\text {distance }}=r_{\text {ion }}+r_{\text {TEA }}\right.$; ionic radius of $1.33 \AA$ for $\mathrm{K}^{+}, 1.48 \AA$ for $\mathrm{Rb}^{+}$, and $1.69 \AA$ for $\mathrm{Cs}^{+}$and the effective radius of $4.5 \AA$ for TEA) $[1,22,23]$. The $\Delta G$ values were calculated from the $K_{\text {occ }}^{\mathrm{TEA}}$ values at $+50 \mathrm{mV}$. The data points were fitted by an electrostatic equation [30, 31], $e^{2} /\left(4 \pi \varepsilon \varepsilon_{\mathrm{r}} r_{\text {distance }}\right)$, and the slope indicateds an apparent dielectric constant $\left(\boldsymbol{\varepsilon}_{\mathrm{r}}=6.5\right)$ of complex dielectric media immersing ion-TEA pair.

tion-impeding site should locate close to bound TEA (i.e., the outermost site of the selectivity filter). If the inactivation-impeding site is the third position, the difference in ion size is smeared at the TEA binding site. In the case of close proximity between bound ion and TEA, how is the electrical repulsion force between closely located TEA and an ion overcome? In a molecular dynamics study, TEA and potassium were located adjacent at the entrance of the pore without inserting a water molecule between them [5]. The interaction free energy was not unrealistically high.

Here, we propose a hypothesis that bound TEA and an ion interact electrostatically. Permeating ions bound in the selectivity filter are solvated by eight carbonyl oxygen atoms [2]. Bound ion in the outermost site of the selectivity filter exposes only a part of its surface to the pore entrance. Thus, TEA can bind to the pore entrance being subjected to the attenuated electrostatic repulsion force from the buried ion in the outermost site. If they are in close contact, the distance between TEA and the ion becomes $r_{\mathrm{TEA}}+r_{\mathrm{ion}}$. In Fig. 5, the free energy of the TEA binding calculated from the $K_{\mathrm{occ}}^{\mathrm{TEA}}$ values (Fig. 3 legends) is plotted as a function of the distance. Here, the slope represents the inverse of the dielectric constant $\left(\varepsilon_{\mathrm{r}}=6.5\right)$, assuming that the difference of the free energy is attributed exclusively to electrostatic interaction. This value represents the environment surrounding an alkali cationTEA pair around the entrance of the selectivity filter.

The vacant site is filled by alkali cations from out- 
side with the selectivity of $\mathrm{Rb}^{+}>\mathrm{Cs}^{+}>\mathrm{K}^{+}$. Thus, the outermost site is neither just a dehydration site nor a site with comparable selectivity for the throughput permeation. By focusing on a single permeation site, a discrepancy between local and overall properties was elucidated. Once bound, an ion contributes a part of the TEA binding site, thus controlling TEA affinity. The characteristics of the outermost site of the selectivity filter elucidated here, based on dynamic measurements, contribute invaluably to further understanding the mechanism of ion permeation and selectivity.

Note added in proof: Since this paper was submitted, a paper has apeared by L. Guidoni and P. Carioni (J Recept Signal Transduct Res 22: 315-331, 2002). They calculated TEA binding for different ion distributions in the selectivity filter of the KcsA potassium channels and exhibited that a quasi-planar form of TEA bound to the $\mathrm{K}-\mathrm{W}-\mathrm{K}-\mathrm{W}$ configuration with only $0.7 \mathrm{kcal} / \mathrm{mol}$ less stable than to the $\mathrm{W}-\mathrm{K}-\mathrm{W}-\mathrm{K}$ configuration.

We thank Professor Gail A. Robertson (Wisconsin, USA) for providing the HERG clone, Olaf S. Andersen (New York, USA), Shin-Ho Chung (Canberra, Australia), and Andy F. James (Bristol, UK) for their suggestions and advice, Toru Takumi for his technical suggestions, Atsumi Hasegawa, Kenichiro Mita, and Ken Kitamura for their technical assistance, and Katsuyuki Yamada and Takashi Konno for their discussions. This work has been supported by CREST of JST (Japan Science and Technology Corporation) and Grants-in-Aid for Scientific Research from the Ministry of Education, Culture, Sports, Science and Technology of Japan (13680737, 13878133).

\section{REFERENCES}

1. Hille B: Ion Channels of Excitable Membranes, 3rd ed., Sinauer Associates Inc., Sunderland, 2001

2. Zhou Y, Morais-Cabral JH, Kaufman A, and MacKinnon $\mathrm{R}$ : Chemistry of ion coordination and hydration revealed by a $\mathrm{K}^{+}$channel-Fab complex at $2.0 \AA$ resolution. Nature 414: 43-48, 2001

3. Morais-Cabral JH, Zhou Y, and MacKinnon R: Energetic optimization of ion conduction rate by the $\mathrm{K}^{+}$selectivity filter. Nature 414: 37-42, 2001

4. Bernéche $S$ and Roux B: Energetics of ion conduction through the $\mathrm{K}^{+}$channel. Nature 414: 73-77, 2001

5. Åvist $\mathrm{J}$ and Luzhkov $\mathrm{V}$ : Ion permeation mechanism of the potassium channel. Nature 404: 881-884, 2000

6. Chung SH, Allen TW, and Kuyucak S: Conducting-state properties of the KcsA potassium channel from molecular and Brownian dynamics simulations. Biophys $\mathrm{J} 82$ : 628-645, 2002

7. Kuyucak S, Andersen OS, and Chung SH: Models of permeation in ion channels. Rep Progress Phys 64: 1427-1472, 2001

8. Shrivastava IH, Tieleman DP, Biggin PC, and Sansom MS: $\mathrm{K}^{+}$versus $\mathrm{Na}^{+}$ions in a $\mathrm{K}$ channel selectivity filter: a simulation study. Biophys J 83: 633-645, 2002

9. Guidoni $L$ and Carloni P: Potassium permeation through the KcsA channel: a density functional study. Biochim Biophys Acta 1563: 1-6, 2002

10. Burykin A, Schutz CN, Villa J, and Warshel A: Simulations of ion current in realistic models of ion channels: the KcsA potassium channel. Proteins 47: 265-280, 2002

11. Smith PL, Baukrowitz T, and Yellen G: The inward rectification mechanism of the HERG cardiac potassium channel. Nature 379: 833-836, 1996

12. Lopez-Barneo J, Hoshi T, Heinemann SH, and Aldrich RW: Effects of external cations and mutations in the pore region on C-type inactivation of Shaker potassium channels. Receptors Channels 1: 61-71, 1993

13. Fan JS, Jiang M, Dun W, McDonald TV, and Tseng GN: Effects of outer mouth mutations on hERG channel function: a comparison with similar mutations in the Shaker channel. Biophys J 76: 3128-3140, 1999

14. Swenson RP Jr and Armstrong $\mathrm{CM}: \mathrm{K}^{+}$channels close more slowly in the presence of external $\mathrm{K}^{+}$and $\mathrm{Rb}^{+}$. Nature 291: 427-429, 1981

15. Baukrowitz $\mathrm{T}$ and Yellen G: Modulation of $\mathrm{K}^{+}$current by frequency and external $\left[\mathrm{K}^{+}\right]$: a tale of two inactivation mechanisms. Neuron 15: 951-960, 1995

16. Starkus JG, Kuschel L, Rayner MD, and Heinemann $\mathrm{SH}$ : Ion conduction through C-type inactivated Shaker channels. J Gen Physiol 110: 539-550, 1997

17. Zou A, Xu QP, and Sanguinetti MC: A mutation in the pore region of HERG $\mathrm{K}^{+}$channels expressed in Xenopus oocytes reduces rectification by shifting the voltage dependence of inactivation. J Physiol (Lond) 509: 129-137, 1998

18. Schonherr R and Heinemann SH: Molecular determinants for activation and inactivation of HERG, a human inward rectifier potassium channel. J Physiol (Lond) 493: 635-642, 1996

19. Wang S, Liu S, Morales MJ, Strauss HC, and Rasmusson RL: A quantitative analysis of the activation and inactivation kinetics of HERG expressed in Xenopus oocytes. J Physiol (Lond) 502: 45-60, 1997

20. Heginbotham $L$ and MacKinnon R: The aromatic binding site for tetraethylammonium ion on potassium channels. Neuron 8: 483-491, 1992

21. Thompson $\mathrm{J}$ and Begenisich $\mathrm{T}$ : Interaction between quaternary ammonium ions in the pore of potassium channels. Evidence against an electrostatic repulsion mechanism. J Gen Physiol 115: 769-782, 2000

22. Crouzy S, Bernéche S, and Roux B: Extracellular blockade of $\mathrm{K}^{+}$channels by TEA: results from molecular dynamics simulations of the KcsA channel. J Gen Physiol 118: 207-218, 2001

23. Luzhkov VB and Åqvist J: Mechanisms of tetraethylammonium ion block in the KcsA potassium channel. FEBS Lett 495: 191-196, 2001

24. Choi KL, Aldrich RW, and Yellen G: Tetraethylammonium blockade distinguishes two inactivation mechanisms in voltage-activated $\mathrm{K}^{+}$channels. Proc Natl 
Acad Sci USA 88: 5092-5095, 1991

25. Trudeau MC, Warmke JW, Ganetzky B, and Robertson GA: HERG, a human inward rectifier in the voltagegated potassium channel family. Science 269: 92-95, 1995

26. Sabirov RZ, Tominaga T, Miwa A, Okada Y, and Oiki S: A conserved arginine residue in the pore region of an inward rectifier K channel (IRK1) as an external barrier for cationic blockers. J Gen Physiol 110: 665-677, 1997

27. Bevington PR: Data Reduction and Error Analysis for the Physical Sciences, McGraw-Hill, Inc, New York, 1969

28. Woodhull AM: lonic blockage of sodium channels in nerve. J Gen Physiol 61: 687-708, 1973

29. Hill TL: Free Energy Transduction and Biochemical Cycle Kinetics, Springer-Verlak, New York, USA, 1988

30. Fersht A: Structure and Mechanism in Protein Science: A Guide to Enzyme Catalysis and Protein Folding, WH Freeman \& Co, New York, 1999

31. Isaraelachvili JN: Intermolecular and Surface Forces, 2nd ed, Academic Press Ltd, London, 1992

\section{APPENDIX}

\section{Inactivation time constant as a function of TEA or potassium concentration}

Inactivation is decelerated by external TEA since the channel can inactivate only if it is unblocked. The inactivation rate becomes $k_{\text {Inact }} p_{\text {open }} /\left(p_{\text {open }}+p_{\text {block }}\right)+$ $k_{\text {DInact }}$, where $p_{\text {open }}$ and $p_{\text {block }}$ are the steady-state open and blocked probabilities. $k_{\text {Inact }}$ and $k_{\text {DInact }}$ are the inactivation and deinactivation rate, respectively. Thus,

$$
\tau^{\mathrm{TEA}}=\frac{1}{k_{\text {Inact }}} \frac{1+[\mathrm{TEA}] / K^{\mathrm{TEA}}}{1+K_{\mathrm{I}}\left(1+[\mathrm{TEA}] / K^{\mathrm{TEA}}\right)}
$$

where $K^{\mathrm{TEA}}$ is the dissociation constant of TEA, and $K_{\mathrm{I}}$ is the equilibrium constant for inactivation $\left(=k_{\text {DInact }} / k_{\text {Inact }}\right)$. When $K_{\mathrm{I}}$ is negligible, the equation becomes linear. The normalized time constant is expressed as follows:

$$
\frac{\tau^{\mathrm{TEA}}}{\tau^{\mathrm{o}}}=1+\frac{[\mathrm{TEA}] / K^{\mathrm{TEA}}}{1+K_{\mathrm{I}}\left(1+[\mathrm{TEA}] / K^{\mathrm{TEA}}\right)}
$$

Permeating potassium impedes inactivation by the foot-in-the-door mechanism. Inactivation proceeds when the outer selectivity filter is vacant [16]. Ion distribution for permeating ions in the selectivity filter is divided into two ensembles of states with respect to the inactivation-impeding site being occupied $\left(\mathrm{O}_{\text {occ }}\right)$ or vacant $\left(\mathrm{O}_{\mathrm{vct}}\right)$. The steady-state approximation for the probability of open states was applied since transitions between open states with different ion distributions are much faster than those between open and inactivated states. The steady-state probability of the oc- cupied-open and vacant-open states can be expressed by the ratio of polynomials of ion concentrations. The order of the polynomial depends on the number of the ion-binding sites for multi-ion permeation. Here, the simplest case, which represents not only one-ion pore but also the shift model deduced from ion distribution in the KcsA potassium channel $[3,5]$, is shown. The inactivation-impeding site is filled by a permeating ion from either an outer or inner site. Thus, the transition from vacant pore to occupied pore was expressed by four rate constants with concentration-dependent (the second-order) and independent (the first-order) factors. The vacant probability becomes $p_{\mathrm{vct}}=p_{\mathrm{vct}}^{\mathrm{o}} /$ $\left(1+[\mathrm{K}] / K^{\mathrm{K}}\right)$, where $p_{\mathrm{vct}}^{\mathrm{o}}$ is the vacant probability in the absence of external potassium and $K^{\mathrm{K}}$ is the dissociation constant of potassium. Therefore, the vacant probability does not become unity even in the absence of external potassium. Ion permeation occurs through the cycle flux around these two states. Thus,

The inactivation rate becomes $k_{\text {Inact }} p_{\text {vct }}+k_{\text {DInact }}$.

$$
\tau^{\mathrm{K}}=\frac{1}{k_{\text {Inact }}^{\mathrm{o}}} \frac{1+[\mathrm{K}] / K^{\mathrm{K}}}{1+K_{\mathrm{I}}\left(1+[\mathrm{K}] / K^{\mathrm{K}}\right)}
$$

The apparent inactivation rate, $k_{\text {Inact }}^{0}\left(=p_{\text {vct }}^{o} \times k_{\text {Inact }}\right)$, is the rate in the absence of external potassium. The normalized time constant is expressed as follows:

$$
\frac{\tau^{\mathrm{K}}}{\tau^{\mathrm{o}}}=1+\frac{[\mathrm{K}] / K^{\mathrm{K}}}{1+K_{\mathrm{I}}\left(1+[\mathrm{K}] / K^{\mathrm{K}}\right)}
$$

\section{The five-state model}

The inhibitory action of TEA and permeating ions on inactivation was expressed as a five-state model (Scheme 3). A formula was derived under the assumptions that (1) the channel immediately reaches the steady-state among the open states (empty and occupied states) and blocked states (vacant-blocked and occupied-blocked states), and (2) inactivation proceeds only from the unblocked vacant pore:

$$
\tau_{\text {Inact }}=\frac{1}{\frac{k_{\text {Inact }}^{\mathrm{o}}}{1+\frac{[\mathrm{TEA}]}{K^{\mathrm{TEA}}}+\frac{[\mathrm{X}]}{K^{\mathrm{Ion}}}+\frac{[\mathrm{TEA}]}{K_{\mathrm{occ}}^{\mathrm{TEA}}} \frac{[\mathrm{X}]}{K^{\text {Ion }}}}+k_{\text {DInact }}}
$$

where $[\mathrm{X}]$ represents the concentration of an alkali cation and the dissociation constant of the $\mathrm{X}$ ion is $K^{\text {Ion }}$. The dissociation constant of TEA to the vacant pore is $K_{\mathrm{vct}}^{\mathrm{TEA}}$ and that to the occupied pore is $K_{\mathrm{occ}}^{\mathrm{TEA}}$. 
The apparent dissociation constant of TEA $\left(K^{\mathrm{TEA}}\right)$ is determined by a combination of these dissociation constants $\left(=\left(1-p_{\mathrm{vct}}^{\mathrm{o}}\right) / K_{\mathrm{occ}}^{\mathrm{TEA}}+p_{\mathrm{vct}}^{\mathrm{o}} / K_{\mathrm{vct}}^{\mathrm{TEA}}\right)$.

From this equation, qualitative features of interaction between inhibitors can be predicted. For example, potassium dependency of the $K^{\mathrm{TEA}}$ value can be expressed as:

$$
K^{\mathrm{TEA}}=\frac{1+\frac{p_{\mathrm{emp}}^{\mathrm{o}}}{K^{\mathrm{Ion}}}[\mathrm{X}]}{\frac{1-p_{\mathrm{emp}}^{\mathrm{o}}}{K_{\mathrm{occ}}^{\mathrm{TEA}}}+\frac{p_{\mathrm{emp}}^{\mathrm{o}}}{K_{\mathrm{emp}}^{\mathrm{TEA}}}+\frac{p_{\mathrm{emp}}^{\mathrm{o}}}{K_{\mathrm{occ}}^{\mathrm{TEA}} K^{\mathrm{Ion}}}[\mathrm{X}]}
$$

Differentiating the equation in terms of the concentration leads to a simple relationship: The increase in $K^{\text {TEA }}$ with increased permeant concentration happens only with $K_{\text {occ }}^{\mathrm{TEA}}>K_{\text {vct }}^{\mathrm{TEA}}$.

When the deinactivation rate is negligible $\left(K_{\mathrm{I}} \cong 0\right.$ or $p_{\text {emp }}^{o} k_{\text {Inact }} \gg k_{\text {DInact }}$, which is the case at positive potentials), Eq. A3 becomes a simplified form:
For fixed concentrations of an ion, [TEA] dependency of $\tau_{\text {Inac }}$ becomes linear. Extrapolating each line for different concentrations of an ion to the negative region discriminates a mode for the inhibitory action between the ion and TEA. If an ion and TEA are competitive, TEA cannot bind to the occupied pore $\left(K_{\text {occ }}^{\mathrm{TEA}}=\infty\right)$, thus, the slope is fixed and the lines are parallel. If they are uncompetitive (TEA can bind to vacant and occupied pores equally $\left(K_{\text {occ }}^{\mathrm{TEA}}=K_{\mathrm{vct}}^{\mathrm{TEA}}=\right.$ $\left.K^{\mathrm{TEA}}\right)$ ), all lines cross on the abscissa axis at $-K^{\mathrm{TEA}}$. If they are neither competitive nor uncompetitive, lines still intersect in the negative region at the coordinate of

$$
\{x, y\}=\left\{-K_{\mathrm{occ}}^{\mathrm{TEA}}, \frac{1}{p_{\mathrm{emp}}^{\mathrm{o}} k_{\text {Inact }}}\left(1-\frac{K_{\mathrm{occ}}^{\mathrm{TEA}}}{K^{\mathrm{TEA}}}\right)\right\} .
$$

The crossing point locates at the third quadrant, if $K_{\mathrm{occ}}^{\mathrm{TEA}}>K^{\mathrm{TEA}}\left(K_{\mathrm{occ}}^{\mathrm{TEA}}>K_{\mathrm{vct}}^{\mathrm{TEA}}\right.$ from Eq. A4).

$$
\tau_{\text {Inact }}=\frac{1}{k_{\text {Inact }}^{\mathrm{o}}}\left\{1+\frac{[\mathrm{X}]}{K^{\mathrm{Ion}}}+\left(\frac{1}{K^{\mathrm{TEA}}}+\frac{1}{K_{\mathrm{occ}}^{\mathrm{TEA}}} \frac{[\mathrm{X}]}{K^{\text {Ion }}}\right)[\mathrm{TEA}]\right\}
$$

